\title{
Parotid Squamous Cell Carcinoma: Is it a Primary or Secondary?
}

\author{
Aboziada A Mohamed and Eisbruch, Avraham. \\ Department of Radiation Oncology University of Michigan, Ann Arbor, MI, USA., Department of \\ radiation Oncology, South Egypt Cancer Institute, Assiut University
}

\begin{abstract}
Background: To evaluate patients with parotid squamous cell carcinoma (SCC) to see is it a primary or metastatic and determine the outcome of patients who metastasis to the parotid.

Patients and Methods: Fifty nine patients were identified with parotid SCC through the hospital data-base, patients' charts, imaging studies and computerized notes.

Results: Fifty patients diagnosed as metastatic SCC of parotid lymph nodes. However, 9 patients diagnosed as primary. Forty seven patients with metastatic SCC treated with surgery and post-operative radiotherapy. The median follow up of these patients was 33 months. The 3-year actuarial cause specific survival was $91 \%$. The 3-year actuarial local recurrence free survival (LRFS) and disease free survival rate were $79 \%$ and $77 \%$ respectively. Positive surgical margin after parotidectomy was the only factor that had statistically significant poor LRFS.

Conclusion: The most common site of the primary tumor was a Cutaneous SCC of the face. All patients should be considered for facial sparing radical parotidectomy and adjuvant radiotherapy. Neck dissection or elective neck irradiation for ipsilateral neck is recommended due to high occult LN metastasis.
\end{abstract}

Key words: Parotid, parotidectomy and radiotherapy.

Corresponding Author: Aboziada A Mohamed

E-mail: maboziada70@yahoo.com

\section{INTRODUCTION}

Parotid squamous cell carcinoma (SCC) is either primary, when another primary site of origin is unknown or when it is not present in parotidean lymph nodes, or metastatic. Primary SCC originating in the parotid is a rare and aggressive tumor, with reported incidence of only $0.3 \%$ to $1.5 \%$. More commonly, SCC is metastatic to the intraparotid and periparotid lymph nodes from ipsilateral cutaneous malignancy of the face and $\operatorname{scalp}^{1,2,3}$. When SCC of the parotid is diagnosed, efforts must be made to identify the primary site. When no primary lesion exists or when the SCC is not confined to parotidean lymph nodes, it seems logical to consider it a primary SCC of the parotid. In both metastatic and primary SCC of the parotid, optimal treatment was unclear ${ }^{4,5}$. This may be due to rarity of the disease; therefore, most of the trials are retrospective and the bias in treatment selection. The policy of treatment in the University of Michigan hospital is facial nerve sparing parotidectomy followed by adjuvant radiotherapy. The aim of this study is to evaluate patients with parotid SCC to see is it a primary or metastatic and determine the outcome of patients who metastasis to the parotid.

\section{PATIENTS AND METHODS}

Patients were retrospectively identified through the hospital data-base, patients' charts, imaging studies and computerized notes, after receiving the approval of Institutional Review Board.

During the period of 1995 to 2008, fifty nine patients diagnosed with parotid SCC were treated in the department of radiation oncology, University of Michigan. Age of patients ranged from 51-91years (Median=73 years). Forty nine patients were female and 10 patients were male. Fifty of the 59 patients were diagnosed as metastatic SCC to intra or peri-parotid lymph nodes. However, 9 patients were diagnosed as primary SCC of the parotid.

Statistical Design: Kaplan-Meier estimates of actuarial survival, disease free survival, local recurrence free survival, were calculated. For comparisons of survival differences, the log-rank test was performed. Cross tabulation for the analyzed parameters were done with Fisher's test; the P-values were double-sided with $\mathrm{p}<0.05$ considered statistically significant.

\section{RESULTS}

Patients' characteristics for all patients are summarized in Table (1). 
Patients with metastatic SCC. Forty eight of the fifty patients with metastatic SCC had a history of primary disease. Of those patients, 47 patients had a history of cutaneous SCC of the face and one patient had a history of SCC of the alveolar mucosa. Two patients had no history of primary lesion, however, one patient developed helix SCC later. The most common sites of cutaneous SCC were the auricle (19 patients) followed by the check (13 patients). Forty one of the fifty patients developed the parotid lesion after a median 7 months of the primary lesion and 9 patients presented with concurrent primary lesion. Of the fifty patients, 8 patients presented with ipsilateral neck lymph nodes in level II and 2 patients had facial palsy. Fourteen patients were immunocompromised; 5 patients had organ transplantation, 6 patients had hematological malignancy and 3 patients had solid malignancy.

All 50 patients underwent a parotidectomy. Thirty four $(68 \%)$ patients had a conservative (facial nerve sparing) parotidectomy. Ten of these patients had positive margins. The remaining $16(32 \%)$ patients had a total parotidectomy with sacrifice of the facial nerve and nine patients were identified as having positive margins. Ipsilateral neck dissection was performed in $44(88 \%)$ patients. Of those 44 patients, 23 patients had selective neck dissection (SND) of level I-III, 20 patients had modified radical neck dissection (MRND) and one patient had radical neck dissection (RND).

Positive margins were found in 19 of 50 patients (38\%) and peri-neural invasion (PNI) in 13 patients (26\%). Nine patients had extra capsular extension (ECE) of parotid L.N with a median 3 L.N pathologically positive (Range: 1-7 L.N). Regarding histological differentiation, 6 patients had grade I, 30 patients had grade II and 14 patients had grade III. The incidence of pathologically positive neck L.N was 54\% (24/44) and 9 of them had ECE. The eight patients, who had clinically positive neck, all had pathological positive disease. Therefore, the incidence of occult cervical metastasis was 44\% (16/36) (Table 1). The most common sites of L.N metastasis were level II, level Va and level III respectively.

Postoperative radiotherapy (RT) was prescribed to 47 patients (94\%) to the parotid region, of whom 42 patients received RT also to the ipsilateral neck and 2 patients received bilateral neck RT. Therefore, 44 patients received post-operative neck irradiation plus parotid region. The dose was $60 \mathrm{~Gy} / 30$ fractions/ 6 weeks for the parotid and positive L.N regions. Lower neck L.N received a prophylactic dose of $50 \mathrm{~Gy} / 25$ fractions $/ 5$ weeks. Areas of positive margins and ECE were boosted to $66 \mathrm{~Gy}$. The majority of patients were treated with 3D conformal RT. Thirteen patients $(27 \%)$ received concurrent chemo-radiation.
Patients with primary SCC. Three of nine patients presented with upper neck L.N and one patient had a facial palsy. No patient was immunocompromised. All patients underwent a total parotidectomy and the facial nerve was preserved in 3 patients. Ipsilateral neck dissection was performed in 7 patients (4 SND, 1 MRND and 2 RND).

Three patients (33\%) had positive margins and PNI had observed in 4 patients (44\%). High grade differentiation was defined in 5 patients $(55 \%)$. Grade II and grade I were observed in 2 patients for each. The median size of the tumor was $4 \mathrm{~cm}$ (Range $1.5-6 \mathrm{~cm}$ ). Three of the 7 patients, who underwent neck dissection, had pathological positive disease and 2 of them had ECE. Level II and III were the sites of L.N metastasis.

All patients (9) received postoperative RT to the parotid and 4 patients received concurrent chemoradiation. Eight patients received ipsilateral neck RT and one patient received bilateral neck RT. The doses of RT were the same as metastatic SCC.

\section{Sites of failure:}

Patients with metastatic SCC. Eleven patients developed parotid region recurrence (Table 2). Three of these patients did not receive postoperative RT. Therefore, the incidence of local failure (LF) was $17 \%$ for the patients who received postoperative RT (8/ 47). No patient developed neck recurrence. Local recurrence developed in a median follow up 16 months (range, 4 to 27 months). Distant metastasis developed in 2 patients to lung, one of them concurrent with local failure.

Patients with primary SCC. No patients had locoregional recurrence, however, one patient developed lung metastasis.

\section{Survival:}

Patients with metastatic SCC who were treated with surgery and postoperative RT (47 patients) were analyzed for survival proportion figures (1\&2). The median follow up was 33 months (range, 10 to 99 months). The 3-year actuarial cause specific survival was $91 \%$ figure (1). The 3-year actuarial local recurrence free survival (LRFS) and disease free survival (DFS) rate were $79 \%$ and $77 \%$ respectively figure(2).

Several variables were analyzed for correlations with LRFS (Table 3). Positive surgical margin after parotidectomy was the only factor that was associated with statistically significant poor outcome on LRFS.

Patients with primary SCC had a median follow up 40 months (range, 18 to 60 months). The 3 -year actuarial cause specific survival and DFS were $100 \%$. 
Table 1: Patients' characteristics.

\begin{tabular}{lcccc}
\hline & \multicolumn{2}{c}{ Metastatic SCC (50) } & \multicolumn{2}{c}{ Primary SCC (9) } \\
\hline & NO. & $\mathbf{( \% )}$ & NO. & (\%) \\
\hline Clinical neck L.N & 8 & $(16)$ & 3 & $(33)$ \\
Facial palsy & 2 & $(3)$ & 1 & $(11)$ \\
Neck dissection & 44 & $(88)$ & 7 & $(77)$ \\
Facial preservation & 34 & $(68)$ & 3 & $(33)$ \\
Positive margins & 19 & $(26)$ & 3 & $(33)$ \\
PNI & 13 & $(12)$ & GI 2 & $(44)$ \\
Grade of disease & GI 6 & GII 2 & $(22)$ \\
& GII 30 & GIII 5 & $(22)$ \\
Pathologic neck L.N & GIII 14 & $3 / 7$ & $(56)$ \\
Occult neck L.N spread & $24 / 44$ & $0 / 4$ & $(42)$ \\
Neck LN ECE & $16 / 36$ & $(28)$ & $2 / 3$ & $0 / 4$ \\
Parotid RT & $9 / 24$ & $(44)$ & 9 & $(66)$ \\
Ipsilateral neck RT & 47 & $(37)$ & 8 & $(100)$ \\
Bilateral neck RT & 42 & $(94)$ & 1 & $(88)$ \\
Chemotherapy & 2 & $(84)$ & 4 & 1 \\
Local recurrence & 13 & $(26)$ & 0 & $(44)$ \\
Neck recurrence & 11 & $(22)$ & 0 &
\end{tabular}

SCC: squamous cell carcinoma, L.N: lymph node, PNI: perineural invasion, ECE: extracapsular extension, RT: radiotherapy.

Table 2: Local recurrence (LR) and Treatment modality.

\begin{tabular}{|c|c|c|c|c|}
\hline \multirow[t]{2}{*}{ Treatment Modality of metastatic SCC } & \multicolumn{2}{|c|}{ Number (50) } & \multicolumn{2}{|c|}{ LR (11) } \\
\hline & NO. & $(\%)$ & NO. & $(\%)$ \\
\hline Surgery alone. & 3 & $(6)$ & $3 / 3$ & $(100)$ \\
\hline Surgery+ Radiotherapy. & 34 & $(68)$ & $5 / 34$ & (14) \\
\hline Surgery + Radiotherapy + Chemotherapy. & 13 & $(26)$ & $3 / 13$ & (23) \\
\hline
\end{tabular}

Table 3: Factors affecting local control.

\begin{tabular}{lc}
\hline Factors & P. value \\
\hline Lymph node presentation & 0.3 \\
Tumor $\geq 3 \mathrm{~cm}$ & 0.1 \\
Facial preservation & 0.8 \\
Margin positive & 0.05 \\
Perineural invasion & 0.08 \\
Extracapsular extension & 0.4 \\
Grade & 0.5 \\
Chemotherapy & 0.2 \\
\hline
\end{tabular}

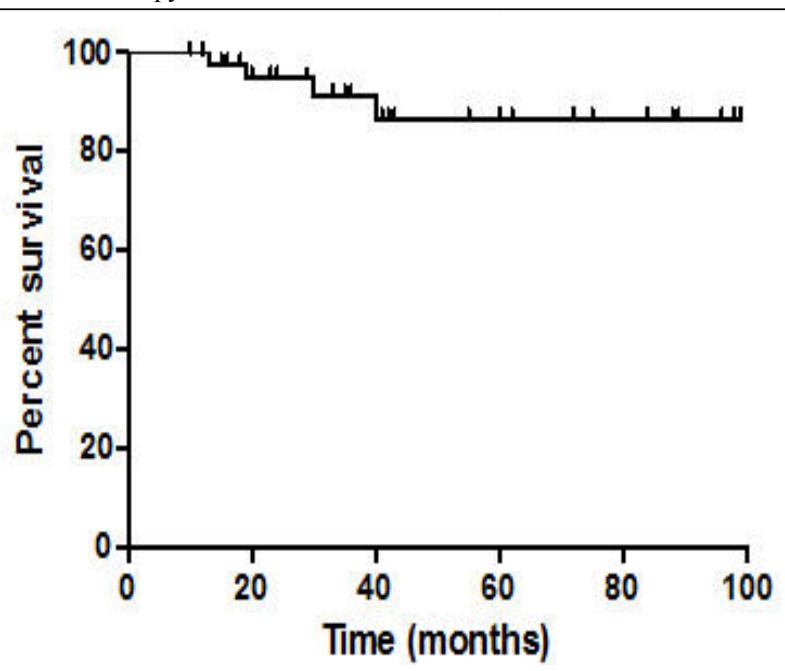

Figure 1: Cause Specific Survival.

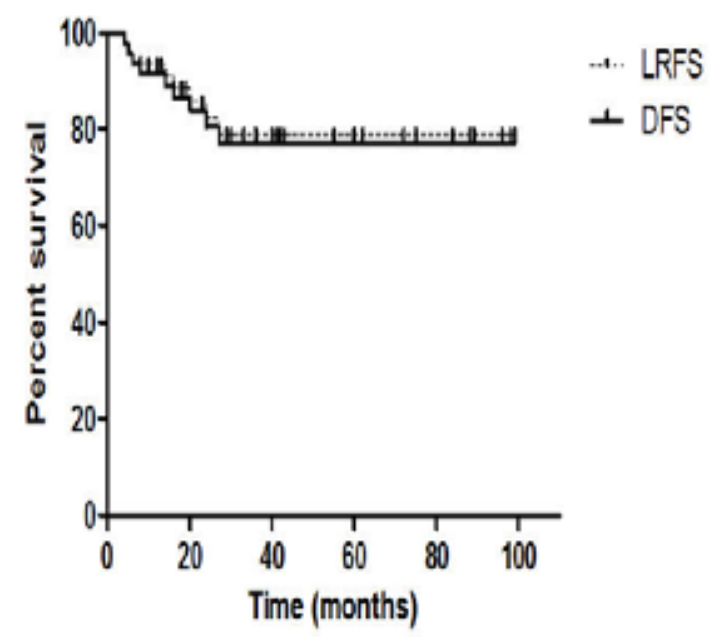

Figure 2: Local Recurrence and Disease Free Survival.

\section{DISCUSSION}

Our data supports previous reports suggesting that parotid SCC is commonly metastasizing from cutaneous SCC. Cutaneous SCC metastasizing to parotid LN is associated with a poor prognosis. Primary parotid SCC represented only $15 \%(9 / 59)$ of all parotid SCC in our 
series and is usually aggressive disease. However, the relatively good outcome of these patients in our series suggests that combined modality treatment, including surgery and adjuvant radiotherapy, have improved the outcome. Publications support surgery and adjuvant $\mathrm{RT}$ as the best practice in resectable tumor ${ }^{6,7,8}$. Our data reported $17 \%(8 / 47)$ local recurrence for patients treated with combined modality. Recent studies reviewed by Veness et al, reported that patients who treated with combined approach can expect $20 \%$ to $25 \%$ chance of local recurrence ${ }^{9}$. Veness et al, designed a prospective trial and reported that RT improved the local control and DFS ( $73 \%$ vs $54 \% ; \mathrm{P}=0.004)$ when compared with surgery alone ${ }^{10}$. In primary parotid SCC, adjuvant radiotherapy is not routine but is usually recommended for high-risk patients, based on grade, stage and margin of excision to improve local control ${ }^{11,12,13}$. In the present study, adjuvant radiotherapy was given to $94 \%$ patients.

The local recurrence rate in our study was $17 \%$, in spite of the combined modality of treatment, high dose of RT and the addition of chemotherapy in $26 \%$ of patients. We studied the influence of some clinico-pathological factors on local recurrence. Positive surgical margin was the only statistically significant factor associated with poor local control $(\mathrm{P}=0.05)$ in this study. Khurana et al. ${ }^{14}$ reported that positive surgical margins were associated with poor local control $(\mathrm{P}=0.02)$, as did O'Brien et al, who identified margin positivity as an independent predictor for parotid recurrence ${ }^{15}$. Several other studies have reported on prognostic factors for predicting local control and survival ${ }^{1,14,16}$. Joseph et al, observed that the involvement of multiple nodes, extracapsular spread, or vascular and perineural invasion were associated with increasing relapse rates ${ }^{1}$. Veness et al. ${ }^{10}$ and Margret et al. ${ }^{17}$, identified extranodal spread as an independent predictor for locoregional recurrence.

Quality of life is an important aim in treatment of parotid tumors. Therefore, facial nerve preservation is important. Extending the surgery to near-total parotidectomy or sacrificing the facial nerve did not seem to make any significant difference in tumor control ${ }^{3,8}$. The extent of surgery is dictated by the extent of tumor, aiming to achieve macroscopic clearance of the tumor and, meanwhile possible, preservation of the facial nerve. Residual microscopic disease is treated by high dose adjuvant radiotherapy. Eddy et al, recommend that the facial nerve is only sacrificed if it is grossly involved by tumor ${ }^{13}$.

Patients with metastatic cutaneous SCC involving the parotid gland had a high incidence of clinical (16\%) and occult neck disease (44\%). Our findings are consistent with Ying et al. $^{3}$ (44\%) and those of O'Brien et al., who reported a $35 \%$ incidence of occult spread in 37 clinically negative necks 5 . Jackson and Ballantyne reported a $24 \%$ rate of occult metastatic SCC and recommended elective treatment of the clinically negativeneck ${ }^{19}$. Researchers from The University of Texas M. D. Anderson Cancer Center documented a $42 \%$ rate of occult metastases ${ }^{20}$. Eddy et al, reported a $16 \%$ incidence of occult spread in 43 clinically negative necks ${ }^{18}$. Treatment of the clinically negative neck should be considered to reduce the likelihood of failure in cervical nodes ${ }^{5,16,18,19}$. Our data identified no neck failure because all patients considered for neck LN treatment. Whether or not surgery ${ }^{14,19}$ or radiotherapy ${ }^{21}$ should be used to treat the clinically negative neck cannot be answered from this study. Elective neck dissection has the benefit of providing additional pathological information with low morbidity and may obviate the need for lower neck irradiation. Patients with clinical involvement of cervical nodes should have a comprehensive neck dissection. Radiotherapy is also delivered to the entire ipsilateral neck if disease is identified in multiple nodes or extracapsular spread in a single node. An undissected ipsilateral neck should be irradiated in the presence of parotid nodal disease even if clinically negative.

A pooled analysis of EORTC ${ }^{22}$ and $\mathrm{RTOG}^{23}$ data in postoperative mucosal head and neck squamous cell carcinoma (HNSCC) demonstrated that combination concurrent platinum chemotherapy and adjuvant radiotherapy improve overall survival and disease-free survival in high-risk patients (extranodal spread and/or positive margin) ${ }^{24}$. Patients with metastatic cutaneous HNSCC with these pathologic features may benefit from means to improve loco-regional control and survival although randomized data in this setting are needed to confirm any hypothesis. From our data, the median age of patients is 73 years. Older patients may tolerate less toxic and more tolerable chemotherapy. Targeted therapy reduces side effects and optimizes treatment. Epidermal growth factor receptor (EGFR) is over expressed in the majority of mucosal HNSCC. Cetuximab inhibits receptor activity by blocking the ligand binding site. It is used as a radiosensitizer and improved the survival and loco-regional control ${ }^{25}$. It could be considered for old age patients with a borderline performance status. This needs a further research for cutaneous HNSCC.

In conclusion, clinicians should be aware of the potential for primary cutaneous SCC of the head and neck to metastasize to parotid lymph nodes. All patients with metastatic SCC should be considered for facial sparing radical parotidectomy and adjuvant radiotherapy. Neck dissection or elective neck irradiation for ipsilateral neck is recommended due to high occult LN metastasis. The addition of chemotherapy or targeted therapy may be beneficial and are points of research. 


\section{REFERENCES}

1. Joseph MG, Zulueta WP, Kennedy PJ. Squamous cell carcinoma of the skin of the trunk and limbs: The incidence of metastases and their outcome. Aust.N.Z.J.Surg. 1992 Sep;62(9):697-701.

2. Czarnecki D, Staples M, Mar A, Giles G, Meehan C. Metastases from squamous cell carcinoma of the skin in Southern Australia. Dermatology 1994;189(1):52-4.

3. Ying YL, Johnson JT, Myers EN. Squamous cell carcinoma of the parotid gland. Head Neck 2006 Jul;28(7):626-32.

4. Toonkel LM, Guha S, Foster P, Dembrow V. Radiotherapy for parotid cancer. Ann.Surg.Oncol. 1994 Nov;1(6):46872.

5. O'Brien CJ, McNeil EB, McMahon JD, Pathak I, Lauer CS. Incidence of cervical node involvement in metastatic cutaneous malignancy involving the parotid gland. Head Neck 2001 Sep;23(9):744-8.

6. Jol JA, van Velthuysen ML, Hilgers FJ, Keus RB, Neering $\mathrm{H}$, Balm AJ. Treatment results of regional metastasis from cutaneous head and neck squamous cell carcinoma. Eur.J.Surg.Oncol. 2003 Feb;29(1):81-6.

7. Veness MJ, Palme CE, Smith M, Cakir B, Morgan GJ, Kalnins I. Cutaneous head and neck squamous cell carcinoma metastatic to cervical lymph nodes (nonparotid): A better outcome with surgery and adjuvant radiotherapy. Laryngoscope 2003 Oct;113(10):1827-33.

8. Bron LP, Traynor SJ, McNeil EB, O'Brien CJ. Primary and metastatic cancer of the parotid: Comparison of clinical behavior in 232 cases. Laryngoscope 2003 Jun;113(6):1070-5.

9. Veness MJ, Porceddu S, Palme CE, Morgan GJ. Cutaneous head and neck squamous cell carcinoma metastatic to parotid and cervical lymph nodes. Head Neck 2007;29(7):621-31.

10. Veness MJ, Morgan GJ, Palme CE, Gebski V. Surgery and adjuvant radiotherapy in patients with cutaneous head and neck squamous cell carcinoma metastatic to lymph nodes: Combined treatment should be considered best practice. Laryngoscope 2005 May;115(5):870-5.

11. Gaughan RK, Olsen KD, Lewis JE. Primary squamous cell carcinoma of the parotid gland. Arch.Otolaryngol. Head.Neck.Surg. 1992 Aug;118(8):798-801.

12. Armstrong JG, Harrison LB, Thaler HT, Friedlander Klar H, Fass DE, Zelefsky MJ, et al. The indications for elective treatment of the neck in cancer of the major salivary glands. Cancer 1992 Feb 1;69(3):615-9.

13. Lee S, Kim GE, Park CS, Choi EC, Yang WI, Lee CG, et al. Primary squamous cell carcinoma of the parotid gland. Am.J.Otolaryngol. 2001 Nov-Dec;22(6):400-6.

14. Khurana VG, Mentis DH, O'Brien CJ, Hurst TL, Stevens GN, Packham NA. Parotid and neck metastases from cutaneous squamous cell carcinoma of the head and neck. Am.J.Surg. 1995 Nov;170(5):446-50.

15. O'Brien CJ, McNeil EB, McMahon JD, Pathak I, Lauer CS, Jackson MA. Significance of clinical stage, extent of surgery and pathologic findings in metastatic cutaneous squamous carcinoma of the parotid gland. Head Neck 2002 May;24(5):417-22.

16. DelCharco JO, Mendenhall WM, Parsons JT, Stringer SP, Cassisi NJ, Mendenhall NP. Carcinoma of the skin metastatic to the parotid area lymph nodes. Head Neck 1998 Aug;20(5):369-73.

17. Chua MS, Veness MJ, Morgan G, Shakespeare T, Hehir A, Gebski V, et al. Parotid lymph-node metastases from cutaneous squamous-cell carcinomas: Treatment outcome and prognostic factors following surgery and adjuvant radiotherapy. Australas.Radiol. 2002 Jun;46(2):174-9.

18. Dona E, Veness MJ, Cakir B, Morgan GJ. Metastatic cutaneous squamous cell carcinoma to the parotid: The role of surgery and adjuvant radiotherapy to achieve best outcome. ANZ J.Surg. 2003 Sep;73(9):692-6.

19. Jackson GL, Ballantyne AJ. Role of parotidectomy for skin cancer of the head and neck. Am.J.Surg. 1981 Oct;142(4):464-9.

20. Moore BA, Weber RS, Prieto V, El Naggar A, Holsinger $\mathrm{FC}$, Zhou X, et al. Lymph node metastases from cutaneous squamous cell carcinoma of the head and neck. Laryngoscope 2005 Sep;115(9):1561-7.

21. Taylor BW,Jr, Brant TA, Mendenhall NP, Mendenhall WM, Cassisi NJ, Stringer SP, et al. Carcinoma of the skin metastatic to parotid area lymph nodes. Head Neck 1991 Sep-Oct;13(5):427-33.

22. Bernier J, Domenge C, Ozsahin M, Matuszewska K, Lefebvre JL, Greiner RH, et al. Postoperative irradiation with or without concomitant chemotherapy for locally advanced head and neck cancer. N.Engl.J.Med. 2004 May 6;350(19):1945-52.

23. Cooper JS, Pajak TF, Forastiere AA, Jacobs J, Campbell $\mathrm{BH}$, Saxman SB, et al. Postoperative concurrent radiotherapy and chemotherapy for high-risk squamouscell carcinoma of the head and neck. N.Engl.J.Med. 2004 May 6;350(19):1937-44.

24. Bernier J, Cooper JS, Pajak TF, van Glabbeke M, Bourhis $\mathrm{J}$, Forastiere A, et al. Defining risk levels in locally advanced head and neck cancers: A comparative analysis of concurrent postoperative radiation plus chemotherapy trials of the EORTC (\#22931) and RTOG (\# 9501). Head Neck 2005 Oct;27(10):843-50.

25. Bonner JA, Harari PM, Giralt J, Azarnia N, Shin DM, Cohen $\mathrm{RB}$, et al. Radiotherapy plus cetuximab for squamous-cell carcinoma of the head and neck. N.Engl.J.Med. $2006 \mathrm{Feb}$ 9;354(6):567-78. 